\title{
Evaluación de la longitud de grietas por fatiga mediante la deformación plástica en muestras compactas de tracción empleando el ruido magnético de Barkhausen $\left(^{(*)}\right.$
}

\author{
F. de los Reyes-Rodríguez*, G. de Diego-Velasco**, J. Capó-Sánchez*** y E. Franco-Fidalgo*
}

\begin{abstract}
Resumen En este trabajo se estudia la influencia de distintos parámetros de la señal del ruido magnético de Barkhausen (RMB) en la deformación plástica (DP) y, en consecuencia, en la longitud de grieta de probetas compactas de tracción (PCT), fabricadas con aceros $12 \mathrm{Cr} 1 \mathrm{MoV}$ y $11 \mathrm{Cr} 1 \mathrm{Mo}$ respectivamente, los cuales son empleados principalmente en la industria termoenergética a nivel mundial. Este estudio se lleva a cabo con el objetivo de conocer, por medio de parámetros del RMB, la longitud de la grieta y el momento más próximo a su incubación, lo cual permite perfeccionar el proceso de preagrietamiento, previo el ensayo de velocidad de propagación de grietas a elevadas temperaturas. Se determina el voltaje medio cuadrado (Vrms) y el máximo voltaje de pico (MVP) de la señal del RMB, magnitudes que decrecen con el incremento de la deformación plástica para cada uno de los aceros estudiados; por otra parte, con el incremento de la deformación plástica se observa, para valores medios de la frecuencia, una correlación lógica entre esta magnitud y la densidad espectral, así como una variación de la amplitud, ancho y forma de los picos de la envolvente de la señal.
\end{abstract}

Palabras clave

Aceros termorresistentes; Deformación plástica; Ruido magnético de Barkhausen; Preagrietamiento; Grieta.

\section{Assessment of fatigue crack length via plastic deformation in compact tension specimens using magnetic Barkhausen noise}

\begin{abstract}
In this paper the influence of different parameters on crack length is determined via plastic deformation using magnetic Barkhausen noise; the compact specimens steel used in this study were $12 \mathrm{Cr} 1 \mathrm{MoV}$ and $11 \mathrm{Cr} 1 \mathrm{Mo}$, which are used for steam transportation on power plants. The main objective of this paper is to determine the crack length and its incubation time using magnetic Barkhausen noise, which lets to improve the pre-cracking process prior creep crack growth rate test simulating the real conditions of high pressure and temperature of main steam lines. Results showed that mean root square and maximum pick of voltage decrease with plastic deformation for each steel analyzed in this work, on the other hand, with the increasing of plastic deformation, a logic relationship between plastic deformation and spectrum density is observed for mid frequencies values, as well as, a variation of the amplitude, width and shape of the Barkhausen signal envelope.
\end{abstract}

Keywords

Heat resistant steel; Plastic deformation; Magnetic Barkhausen noise; Pre-cracking; Crack.

\section{INTRODUCCIÓN}

Los aceros al CrMo y al CrMoV son empleados en gran cantidad de aplicaciones industriales, entre las que se destacan las tuberías para transporte de vapor, fabricación de elementos de sujeción y apoyo en calderas de plantas termoeléctricas entre otras ${ }^{[1-3]}$. De forma general, estos aceros contienen una estructura inicial de ferrita y perlita o ferrita y bainita, que se logra con el tratamiento térmico a temperaturas entre $550{ }^{\circ} \mathrm{C}$ y $700{ }^{\circ} \mathrm{C}$ durante varias horas, el cual genera la precipitación de carburos aleados estables y compuestos intermetálicos, que interfieren en el progreso de las dislocaciones ${ }^{[4]}$. Estos regímenes de calenta-

(•) Trabajo recibido el día 28 de noviembre de 2012 y aceptado en su forma final el día 9 de abril de 2013.

* Dpto. de Manufactura y Materiales, Facultad de Ingeniería Mecánica, Universidad de Oriente, Avda. Patricio Lumumba s/n, CP 90500 , Santiago de Cuba, Cuba.

** Centro de Investigaciones Energéticas Medioambientales y Tecnológicas (CIEMAT), Avda. Complutense 40 (Edf. 30 ), 28040 Madrid, España.

*** Dpto. de Física, Facultad de Ciencias Naturales, Universidad de Oriente, Avda. Patricio Lumumba s/n, CP 90500, Santiago de Cuba, Cuba. 
miento, eventualmente determinan la estabilidad de las propiedades mecánicas de los aceros empleados en plantas térmicas; por ejemplo: la resistencia a la deformación por termofluencia y otras propiedades, así como el tiempo de vida remanente.

El vapor que se transporta en tuberías fabricadas con estos aceros está sometido a temperaturas entre $500{ }^{\circ} \mathrm{C}$ y $560{ }^{\circ} \mathrm{C}$, y provoca en su interior presiones entre $10 \mathrm{MPa}$ y $15 \mathrm{MPa}$. Una vez transcurridos elevados tiempos de explotación a regímenes de termofluencia, la microestructura de estos aceros cambia, la perlita/bainita se descompone, y comienza la precipitación, crecimiento y migración de carburos hacia el límite de los granos de ferrita ${ }^{[5]}$. Este cambio hacia una estructura termodinámicamente inestable, provoca la formación de cavidades y el surgimiento de daños internos, lo cual conduce al fallo del material debido a la estrecha relación que existe entre cambios microestructurales y deterioro de las propiedades mecánicas.

Estos aceros han recibido gran parte de la atención de la comunidad científica a nivel internacional, debido a la importancia económica y social que requiere el estudio de su durabilidad; por ello, entre los principales ensayos que se llevan a cabo se encuentra el de velocidad de propagación de grietas a elevadas cargas y temperaturas, simulando las condiciones reales de explotación ${ }^{[6-9]}$.

A fin de conocer con qué velocidad se propagan las grietas se emplean instalaciones especiales, en las cuales se fijan las variables principales (carga y temperatura). Para el desarrollo de esta técnica se utilizan probetas compactas de tracción (PCT) que se calientan hasta la temperatura de ensayo y se someten a condiciones de cargas estáticas o casi estáticas, es decir, constantes en el tiempo. Los valores del tiempo y longitud de grieta son extraídos del ensayo y procesados, para finalmente obtener la razón de propagación.

Para las PCT no existen requerimientos específicos acerca de su tamaño, parámetro que debe ser escogido en consideración a la geometría del sistema de cargas y horno de ensayo, de forma tal que tenga tamaño suficiente para hacer crecer la grieta y recoger información sobre su longitud.

Para llevar a cabo este experimento se utilizan diferentes normas, entre las que se destaca la ASTM E1457-00 standard test method for measurement of creep crack growth rates in metals ${ }^{[10]}$, la cual exige la presencia de una pregrieta por fatiga, previo el ensayo de velocidad de propagación de grietas a elevadas temperaturas.

Esta pregrieta se obtendrá variando cíclicamente la carga, desde un valor mínimo hasta un valor máximo, la cual será disminuida una vez incubada la grieta para evitar grandes tensiones plásticas por el frente de ésta y tener un mejor control de su crecimiento.
Una de las problemáticas del proceso de preagrietamiento es la dificultad de controlar la longitud de grieta ${ }^{[11]}$; esta operación, por regla general, se lleva a cabo mediante el empleo de strength gages, o desmontando la muestra para su medición en microscopios ópticos; sin embargo, de esta forma no se puede conocer el momento justo de su incubación.

Por tanto, el objetivo principal de este estudio consiste en potenciar, con el efecto Barkhausen, el desarrollo de un método para aplicar in situ, a fin de conocer la longitud de la grieta por fatiga de la PCT, y su momento de incubación, sin necesidad de desmontarla del dispositivo de preagrietamiento para su medición en el microscopio óptico, lo cual, además de las desventajas mencionadas, independientemente de evitar pérdida de tiempo, garantiza que los procesos de remontaje posmedición no afecten el equilibrio inicial de aplicación de cargas para un crecimiento balanceado de la grieta por ambas caras de la PCT.

El método de Barkhausen ${ }^{[12]}$ ha sido empleado para evaluar, tanto la variación de parámetros microestructurales: tamaño de grano ${ }^{[13}$ y 14], porcentaje de carbono $^{[15]}$, carburos $^{[16]}$, contenido de cementita ${ }^{[17]}$, como el efecto de las tensiones aplicadas, la deformación plástica ${ }^{[18]}$, entre otros.

En este trabajo se evalúan magnitudes que se utilizan para caracterizar el RMB, como son: el voltaje medio cuadrado (Vrms) y el máximo voltaje de pico de la señal (MVP), la densidad espectral (DE) y la envolvente; todos estos parámetros son procesados a fin de ser relacionados con la deformación plástica de la entalla de la PCT y posteriormente con la longitud de grieta por fatiga.

\section{MATERIALES Y MÉTODOS}

Las probetas empleadas, como ya se mencionó, son del tipo compactas de tracción (PCT), con dimensión entre el centro de los agujeros y borde posterior (W = 17,5 mm), según norma ASTM E1457-00 [10], fabricadas con aceros $12 \mathrm{Cr} 1 \mathrm{MoV}$ y $11 \mathrm{Cr} 1 \mathrm{Mo}$, empleados en la industria termoenergética para el transporte de vapor sobrecalentado; la composición química de éstos se muestra en la tabla I.

\subsection{Instalación experimental}

En la figura 1 se muestra la instalación experimental utilizada para desarrollar las mediciones de los parámetros del ruido magnético Barkhausen. Ésta emplea una computadora que proporciona ondas sinusoidales de $10 \mathrm{~Hz}$, las cuales son amplificadas por una fuente bipolar que alimenta un circuito con un yoke mag- 

ASSESSMENT OF FATIGUE CRACK LENGTH VIA PLASTIC DEFORMATION IN COMPACT TENSION SPECIMENS USING MAGNETIC BARKHAUSEN NOISE

Tabla I. Composición química de los aceros $12 \mathrm{Cr} 1 \mathrm{MoV}$ y $11 \mathrm{Cr} 1 \mathrm{Mo}$ (\% en peso)

Table I. Chemical composition of 12Cr1MoV and 11Cr1Mo steels (wt \%)

\begin{tabular}{cccccccc}
\hline \multicolumn{7}{c}{ 12Cr1MoV } \\
\hline $\mathbf{C}$ & Si & Mn & $\mathbf{P}$ & $\mathbf{S}$ & $\mathbf{C r}$ & Mo & $\mathbf{V}$ \\
\hline 0,12 & 0,23 & 0,54 & 0,015 & 0,019 & 1,1 & 0,26 & 0,17 \\
\hline \multicolumn{7}{c}{ 11Cr1Mo } \\
\hline 0,21 & 0,36 & 0,49 & 0,011 & 0,009 & 11,15 & 0,85 & 0,28 \\
\hline
\end{tabular}

nético que magnetiza la muestra. Las emisiones magnéticas de Barkhausen son detectadas con un sensor magnético; posteriormente se amplifican y filtran en el orden de $1-150 \mathrm{kHz}$. Concluido este proceso, se visualizan con ayuda de un osciloscopio digital y se capturan empleando una tarjeta de adquisición de datos con frecuencia de muestreo de $200 \mathrm{MHz}$.

Para el estudio de la frecuencia de incubación de grietas y sus longitudes, se midió la deformación plástica en la entalla de la muestra mediante un microscopio óptico, marca Novel, con ocular graduado, el cual fue calibrado con un milímetro internacional.

Para el preagrietamiento de las PCT se utilizó un dispositivo que se adapta a una máquina universal de fatiga por impacto, el cual le transmite cargas cíclicas entre 20 kgf y 150 kgf empleando un muelle helicoidal.

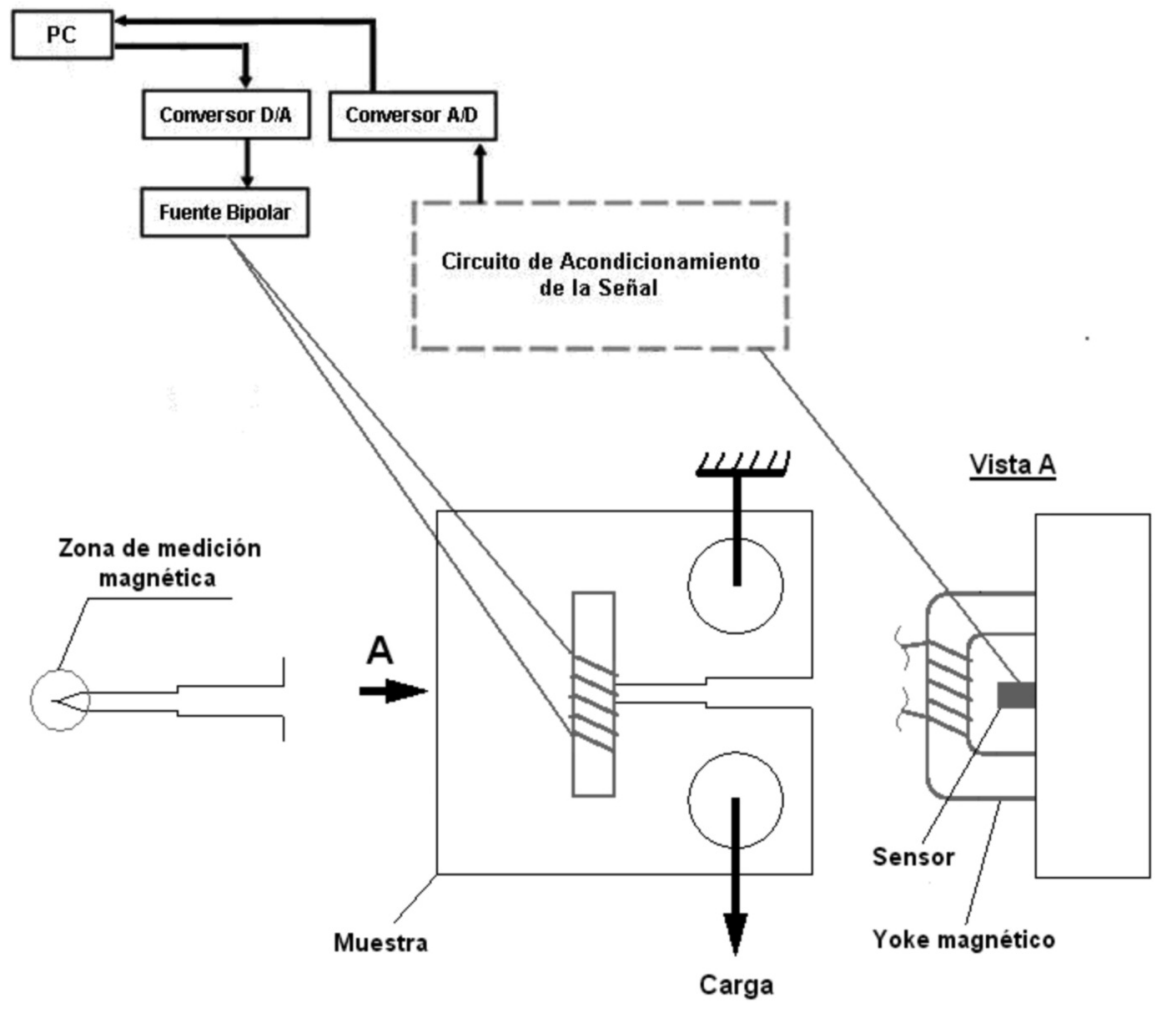

Figura 1. Configuración esquemática del sistema de medición del RMB empleado.

Figure 1. Schematic configuration of the MBN measuring system used. 


\section{RESULTADOS Y DISCUSIÓN}

Como se apuntó anteriormente, existe una relación directa entre la deformación plástica que sufre la muestra compacta de tracción y su longitud de grieta; luego, al obtener una correlación entre los parámetros del RMB y la deformación plástica, se puede lograr, en consecuencia, la correlación entre dichos parámetros y la longitud de la grieta por fatiga que tiene lugar en la muestra analizada.

Todo ello requiere realizar un estudio minucioso de los parámetros del RMB con la deformación plástica de la muestra compacta de tracción. Con este propósito, se evaluaron los parámetros magnéticos para distintos porcentajes de deformación plástica de la entalla de la muestra, y se analizaron los resultados obtenidos, tanto para grandes como para pequeños porcentajes de deformación plástica, lo cual permite, en el primer caso, conocer las longitudes de grieta por fatiga, y en el segundo, el momento en que ésta se incuba.

Varios trabajos sobre el ruido magnético de Barkhausen han demostrado que la tensión aplicada dentro del régimen elástico del material produce efectos en la señal del RMB, que difieren de los que provoca la tensión, más allá del límite elástico, o sea, en el régimen plástico ${ }^{[19]}$.

La deformación plástica en los aceros, de manera general, produce una disminución de la actividad del $\mathrm{RMB}^{[19}$ y 20$]$. Para deformaciones plásticas muy pequeñas, se corroboró un pequeño incremento de la actividad del RMB, lo que coincide con los resultados obtenidos por Stupakov et al. ${ }^{[21]}$. Sobre esta base, en el presente trabajo se logran resultados acerca del

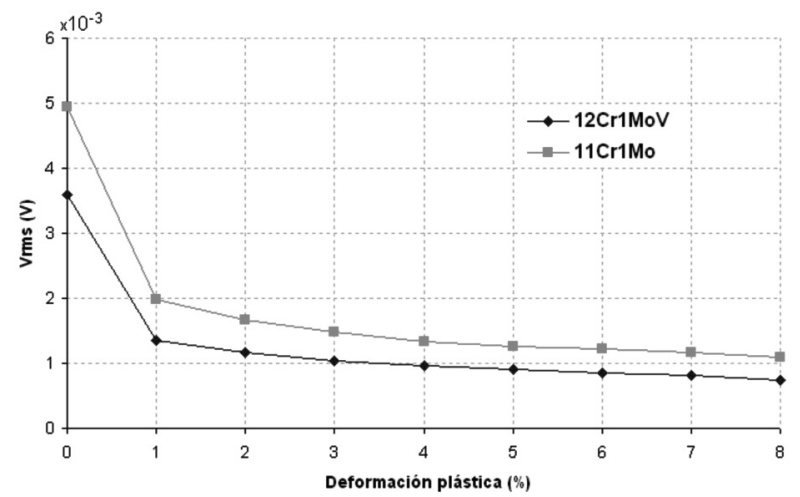

Figura 2. Dependencia del RMB (Vrms) con la deformación plástica: aceros $12 \mathrm{Cr} 1 \mathrm{MoV}$ y $11 \mathrm{Cr} 1 \mathrm{Mo}$.

Figure 2. Dependence of MBN (Vrms) with plastic deformation for $12 \mathrm{Cr} 1 \mathrm{MoV}$ and $11 \mathrm{Cr} 1 \mathrm{Mo}$ steels. comportamiento de parámetros del RMB con diferentes niveles de deformación plástica de las PCT, que son empleados para evaluar la longitud de la grieta por obtener, previo el ensayo de velocidad de crecimiento de grietas a elevadas temperaturas. En la zona de medición magnética de las muestras, mostrada en la figura 1, con el aumento de la deformación plástica se altera el comportamiento magnético debido a los efectos microestructurales que varían con la deformación plástica, los cuales se pueden resumir de la forma siguiente:

- Incremento del número de sitios de anclaje en forma de entramados de dislocaciones, los cuales representan obstáculos al movimiento de las paredes de dominio.

- Desarrollo de una textura cristalográfica que altera la dirección del eje de fácil magnetización.

- Aparición de tensiones residuales locales situadas en los bordes de grano y alrededor de las dislocaciones.

Basado en esto, en la figura 2 se muestra la dependencia entre el voltaje medio cuadrado (Vrms) del RMB y la deformación plástica hasta valores del $8 \%$, que es el límite necesario, en las muestras compactas empleadas, para hacer crecer la grieta hasta el rango de longitud estandarizada entre $1,74 \mathrm{~mm}$ y $1,76 \mathrm{~mm}$, previo el ensayo de velocidad de propagación de grietas a elevadas temperaturas, según norma ASTM E1457-00 [10].

En esta gráfica se muestra el comportamiento de Vrms vs DP de forma general, obviando lo que sucede con deformaciones plásticas inferiores al $1 \%$, lo cual, por su importancia en este trabajo, se analiza con posterioridad. En esta figura se puede apreciar, en el intervalo de deformaciones estudiado, que el Vrms decrece con el incremento de la deformación plástica. Este proceso se atribuye, principalmente, al incremento del número de dislocaciones y a la formación de mallas de éstas ${ }^{[22]}$, las cuales representan grandes obstáculos para el desplazamiento de las paredes de dominio, lo que impide su movimiento. Este resultado concuerda con los obtenidos por Stupakov ${ }^{[23]}$, quien demuestra que para mayores niveles de deformación, el material no llega a saturarse por completo, incluso para altas intensidades de campos magnéticos aplicados; esto evidencia que las paredes de dominio están fuertemente ancladas, e impiden que se sature el material.

Por otra parte, debido a la necesidad de conocer el momento más próximo a la incubación de la grieta (el cual, para los aceros estudiados, surge con deformaciones plásticas en el intervalo de 0,75\% al $1 \%$, según muestra la figura 3), se han llevado a cabo estudios de la correlación de los parámetros del RMB para pequeñas deformaciones plásticas, donde se perciben comportamientos diferentes por la presencia 
de otros fenómenos más complejos, lo que ha sido objeto de estudio por otros investigadores ${ }^{[19]}$.

La conclusión fundamental obtenida del análisis de la figura 3, obliga al estudio de los parámetros del RMB para pequeñas deformaciones plásticas, con el objetivo de conocer in situ en qué momento se incuba la grieta sin necesidad de desmontar la muestra del dispositivo de preagrietamiento.

Para tal propósito, se obtuvo nuevamente la dependencia del Vrms y del máximo voltaje de pico del RMB con la deformación plástica, enfatizando en el comportamiento para deformaciones plásticas inferiores al $1 \%$. Estas dependencias se muestran en las figuras 4 a) y 4 b).

En estas figuras se puede apreciar que la señal del $\mathrm{RMB}$, igualmente decrece para estos niveles de deformación; sin embargo, para deformaciones menores a $1 \%$ ocurre un incremento de la señal. Este fenómeno puede atribuirse al surgimiento ocasional de tensiones residuales en los bordes de granos, las cuales favorecen -en lugar de entorpecer- el movimiento de los dominios de $90^{\circ}$, que son muy sensibles a la tensión ${ }^{[24]}$. Por otra parte, con el incremento de la densidad de las dislocaciones, la estructura de dominios se refina y se forman numerosas paredes de $90^{\circ}$, las cuales, por igual, son muy sensibles a la tensión. Por tanto, cuando existen tensiones residuales en el material, los dominios magnéticos presentan gran movilidad y, debido a que su número es elevado, se produce un incremento de la señal del RMB. Este proceso desaparece posteriormente ya que el gran número de dislocaciones que surgen ocasionadas por la conti-

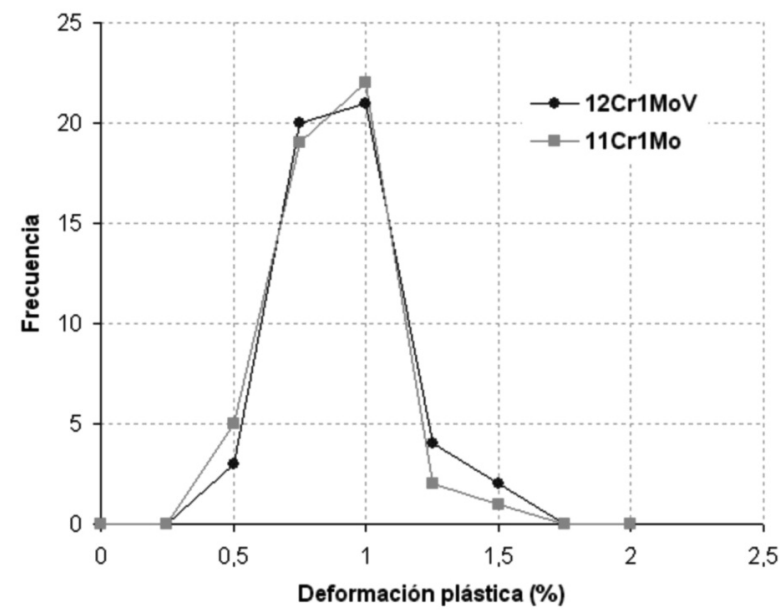

Figura 3. Frecuencia de incubación de grietas por fatiga en los aceros $12 \mathrm{Cr} 1 \mathrm{MoV}$ y $11 \mathrm{Cr} 1 \mathrm{Mo}$.

Figure 3. Fatigue crack incubation frequency in $12 \mathrm{Cr} 1 \mathrm{MoV}$ and $11 \mathrm{Cr} 1 \mathrm{Mo}$ steels.

nuidad de la deformación plástica, impiden el movimiento de las paredes de $90^{\circ}$.

Para estudiar con mayor profundidad la complejidad de la variación de la señal del RMB con pequeñas deformaciones plásticas y, en consecuencia, poder conocer el momento más cercano a la incubación de la grieta, se desarrolla el análisis de la densidad espectral (DE) de la señal del RMB para valores de deformación plástica entre $0 \%$ y $4 \%$ (Figs. 5 a) y 5 b)).

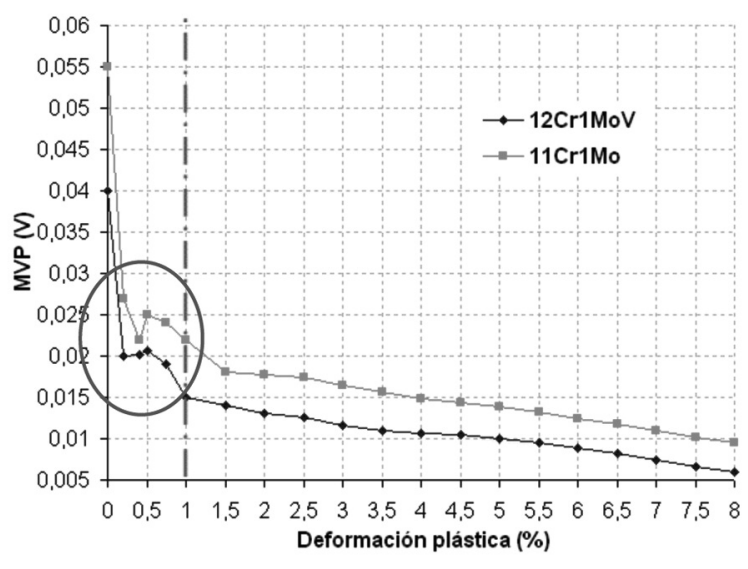

a)

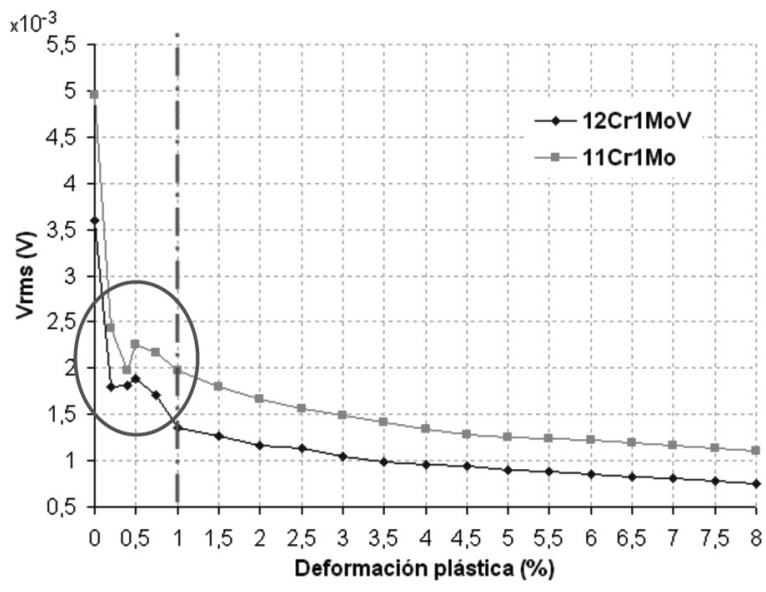

b)

Figura 4. Dependencia del RMB con la deformación plástica en los aceros $12 \mathrm{Cr} 1 \mathrm{MoV}$ y $11 \mathrm{Cr} 1 \mathrm{Mo}$, enfatizando deformaciones inferiores al $1 \%$ : a) MVP y b) Vrms.

Figure 4. Dependence of MBN with plastic deformation in 12Cr1MoV and 11Cr1Mo steels, emphasizing in deformations less than $1 \%$ : a) MVP and b) Vrms. 


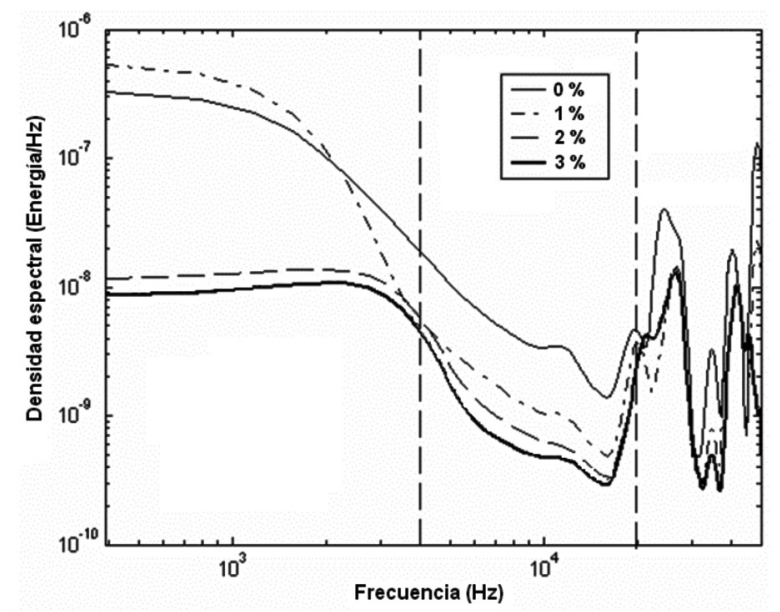

a)

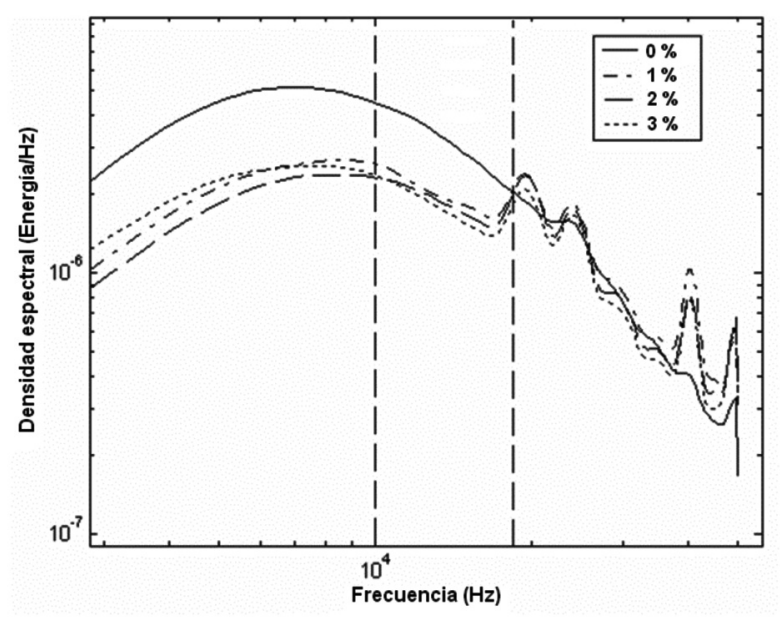

b)

Figura 5. Dependencia de la densidad espectral del RMB con la frecuencia para diferentes niveles de deformación plástica en los aceros: a) $12 \mathrm{Cr} 1 \mathrm{MoV}$ y b) $11 \mathrm{Cr} 1 \mathrm{Mo}$.

Figure 5. Dependence of MBN spectrum density with frequency for different levels of plastic deformation in steels: a) $12 \mathrm{Cr} 1 \mathrm{MoV}$ and b) $11 \mathrm{Cr} 1 \mathrm{Mo}$.

Estas figuras muestran que para bajas y altas frecuencias no hay una dependencia entre la DE y la deformación plástica. Sin embargo, para valores medios de la frecuencia existe una correlación lógica evidente entre ambas. Este fenómeno pudiera atribuirse a que cuando una muestra de acero es sometida a una deformación plástica uniaxial (como es el caso), la tensión presente en la superficie de la muestra difiere de la tensión en el volumen interior, lo cual resulta en una tensión residual en las capas superficiales, y al existir una correlación entre la frecuencia de la señal del RMB y la profundidad desde donde es emitida ${ }^{[25}$ y 26], cuanto mayor es la frecuencia de la señal, más cercana a la superficie ocurren las emisiones del RMB. Las frecuencias muy altas medidas en la DE están influidas por elementos superficiales como la rugosidad, por eso no presentan una dependencia evidente con la deformación. La región intermedia representa la superficie inmediata inferior donde existe una correlación clara de la señal del $\mathrm{RMB}$ con la deformación: la señal del RMB decrece con la deformación plástica, y para menores frecuencias se obtiene información de todo el material, pues las bajas frecuencias proceden tanto de la superficie como del volumen interior. Esta parte de la DE, por tanto, contiene información de varias regiones cuyas correlaciones con la deformación son distintas, lo cual da como resultado que la correlación del RMB con la deformación para bajas frecuencias no sea evidente; estos resultados coinciden con los obtenidos por Moorthy y colaboradores ${ }^{[27]}$.

Por otra parte, se llevó a cabo el análisis de las envolventes de la señal para DP de hasta el $3 \%$, las cuales se muestran en las figuras 6 a) y 6 b) para los aceros $12 \mathrm{Cr} 1 \mathrm{MoV}$ y $11 \mathrm{Cr} 1 \mathrm{Mo}$, respectivamente.

En estas figuras se puede apreciar que la envolvente del RMB para estos niveles de deformación plástica sufre las siguientes modificaciones con el incremento de la deformación:

a) La amplitud de la envolvente disminuye con la deformación plástica.

b) El ancho de la envolvente crece con la deformación plástica.

c) La envolvente tiende a dividirse en dos picos, los cuales van disminuyendo de amplitud hasta que prácticamente desaparecen.

Esto último se puede atribuir de nuevo a los efectos microestructurales que varían con la deformación plástica, los cuales se manifiestan como un incremento en la cantidad de dislocaciones y tensiones residuales. El incremento de las dislocaciones impide el movimiento tanto de las paredes de $180^{\circ}$, como el de las paredes de $90^{\circ}$; éstas se encuentran tan fuertemente ancladas que se dificulta su movimiento y ello provoca que los picos desaparezcan.

En la figura 7 se muestra la correlación existente entre la deformación plástica de la entalla de la muestra compacta de tracción y las longitudes de grieta que crecen como resultado de la aplicación de cargas cíclicas.

Como se comentó anteriormente, se conoce que la grieta, de forma general en los aceros estudiados, se incuba con deformaciones plásticas entre 0,75\% y $1 \%$; por tanto, para evaluar el crecimiento de la grieta con los parámetros del RMB, se obtienen las ecuaciones del modelo de deformación plástica (DP) y longitud de grieta $(\mathrm{Lg})$ (mostrado en la figura 7) y los de deformación plástica, MVP y Vrms respecti- 


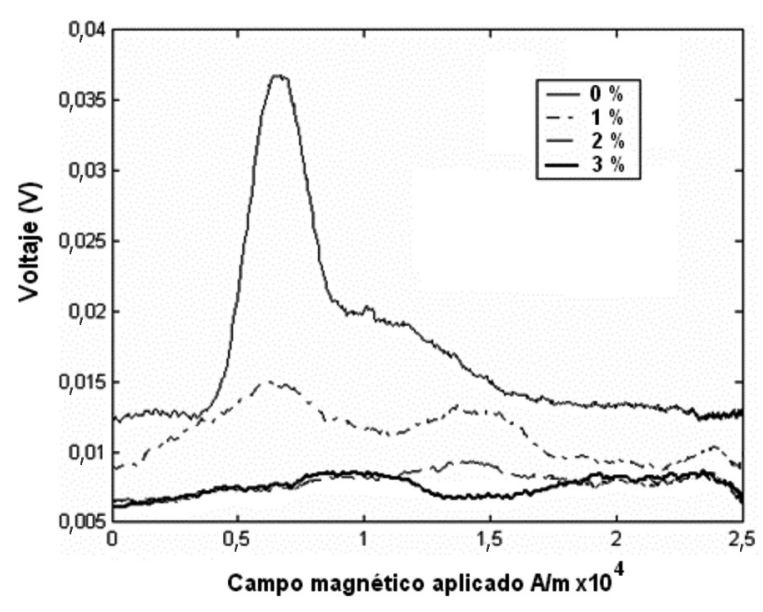

a)

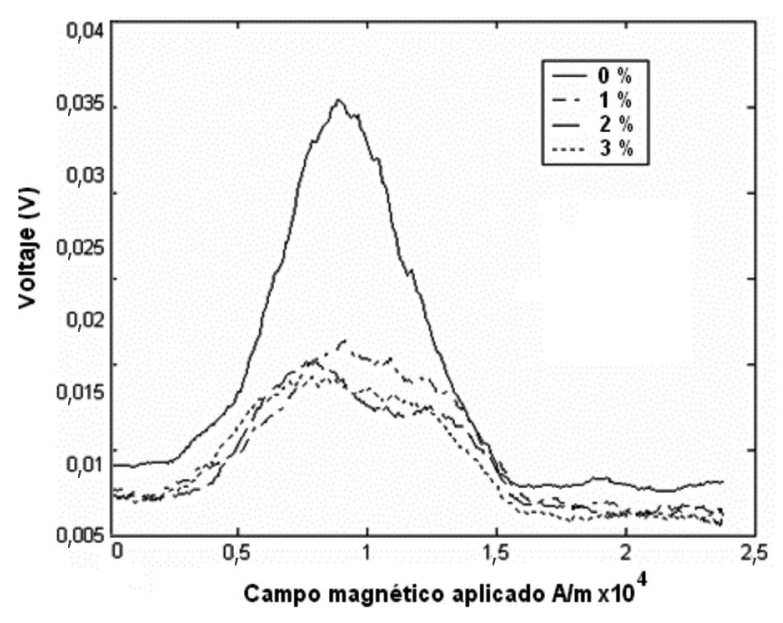

b)

Figura 6. Dependencia de la envolvente del RMB con la deformación plástica para los aceros: a) $12 \mathrm{Cr} 1 \mathrm{MoV}$ y b) $11 \mathrm{Cr} 1 \mathrm{Mo}$.

Figure 6. Dependence of MBN envelop with plastic deformation for steels: a) $12 \mathrm{Cr} 1 \mathrm{MoV}$ and b) $11 \mathrm{Cr} 1 \mathrm{Mo}$.

vamente (mostrados en las figuras 4 a) y 4 b)) los cuales han sido construidos a partir de los valores del rango de deformación plástica entre el $1 \%$ y el $8 \%$, que son las deformaciones plásticas necesarias para hacer crecer la grieta por fatiga hasta su longitud estandarizada. Dichos modelos se muestran en las siguientes ecuaciones:

$$
\operatorname{Lg}=0,23 D P-0,0951
$$

- Para el acero $12 \mathrm{Cr} 1 \mathrm{MoV}$ :

$$
\text { Vrms }=0,0014-0,00029 \ln (D P)
$$

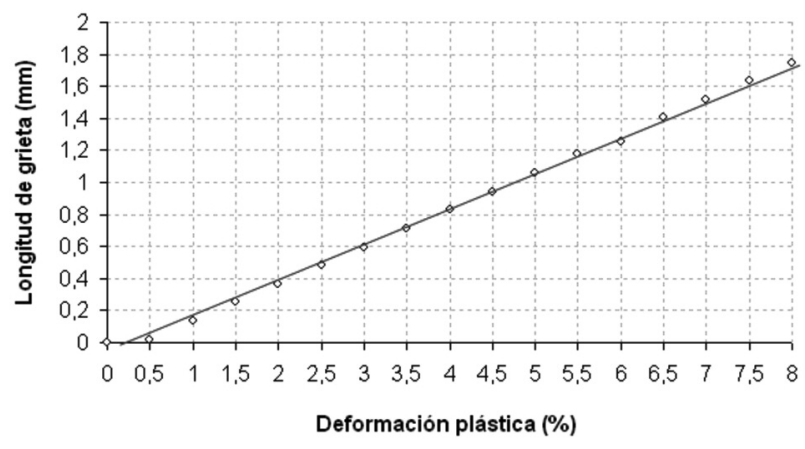

Figura 7. Dependencia entre la deformación plástica y la longitud de grieta en muestras compactas de tracción en los aceros $12 \mathrm{Cr} 1 \mathrm{MoV}$ y $11 \mathrm{Cr} 1 \mathrm{Mo}$.

Figure 7. Dependence between plastic deformation and crack length in compact specimens in $12 \mathrm{Cr} 1 \mathrm{MoV}$ and $11 \mathrm{Cr} 1 \mathrm{Mo}$ steels.

- Para el acero 11Cr1Mo:

$$
\text { Vrms }=0,002-0,00042 \ln (\mathrm{DP})
$$

Despejando DP de las ecuaciones (1), (2) y (3), e igualando este término, se obtiene:

- Para el acero $12 \mathrm{Cr} 1 \mathrm{MoV}$ :

$$
\operatorname{Lg}=0,23\left(e^{\frac{0,0014-v r m s}{0,00029}}\right)-0,0951
$$

- Para el acero 11Cr1Mo:

$$
\operatorname{Lg}=0,23\left(\mathrm{e}^{\frac{0,002-\mathrm{vrms}}{0,00042}}\right)-0,0951
$$

Para el caso del máximo voltaje de pico, se desarrolla el mismo procedimiento:

- Para el acero $12 \mathrm{Cr} 1 \mathrm{MoV}$ :

$$
\mathrm{MVP}=0,016-0,0012(\mathrm{DP})
$$

- Para el acero 11Cr1Mo:

$$
M V P=e^{(-3,78-0,105 D P)}
$$

Despejando DP de las ecuaciones (1), (6) y (7), e igualando este término, se obtiene:

- Para el acero $12 \mathrm{Cr} 1 \mathrm{MoV}$ :

$$
\operatorname{Lg}=\frac{0,2301(0,016-M V P)}{0,0012}-0,0951
$$


- Para el acero 11Cr1Mo:

$$
\operatorname{Lg}=\frac{0,2301(-3,78-\operatorname{LN}(\mathrm{MVP}))}{0,105}-0,0951
$$

Con las expresiones (4), (5), (8) y (9) se pueden obtener, a partir del Vrms y MVP, las longitudes de grieta por fatiga, en muestras compactas de tracción fabricadas con acero $12 \mathrm{Cr} 1 \mathrm{MoV}$ y $11 \mathrm{Cr} 1 \mathrm{Mo}$, respectivamente.

En las tablas II y III se muestran, a partir de las ecuaciones obtenidas, los valores del Vrms y MVP para distintas longitudes de grieta por fatiga en muestras compactas de tracción, para los aceros $12 \mathrm{Cr} 1 \mathrm{MoV}$ y $11 \mathrm{Cr} 1 \mathrm{Mo}$ respectivamente.

Tabla II. Valores de Vrms para distintas longitudes de grieta por fatiga en muestras compactas de tracción

Table II. Vrms values for different fatigue crack length in compact specimens

\begin{tabular}{lclccccc}
\hline \multicolumn{3}{c}{ Acero 12Cr1MoV } & \multicolumn{5}{c}{ Acero 11Cr1Mo } \\
\hline Vrms (V) & Lg (mm) & Vrms (V) & Lg (mm) & Vrms (V) & Lg (mm) & Vrms (V) & Lg (mm) \\
\hline 0,0015 & 0,0678 & 0,001 & 0,8185 & 0,0023 & 0,0175 & 0,0013 & 1,1226 \\
0,00145 & 0,0985 & 0,00095 & 0,9904 & 0,0022 & 0,0478 & 0,0012 & 1,4499 \\
0,0014 & 0,1349 & 0,0009 & 1,1946 & 0,0021 & 0,0862 & 0,00119 & 1,4871 \\
0,00135 & 0,1782 & 0,00085 & 1,4373 & 0,002 & 0,1349 & 0,00118 & 1,5253 \\
0,0013 & 0,2296 & 0,00084 & 1,4910 & 0,0019 & 0,1967 & 0,00117 & 1,5643 \\
0,00125 & 0,2907 & 0,00083 & 1,5467 & 0,0018 & 0,2752 & 0,00116 & 1,6043 \\
0,0012 & 0,3633 & 0,00082 & 1,6043 & 0,0017 & 0,3747 & 0,00115 & 1,6452 \\
0,00115 & 0,4495 & 0,00081 & 1,6639 & 0,0016 & 0,5010 & 0,00114 & 1,6872 \\
0,0011 & 0,5520 & 0,0008 & 1,7256 & 0,0015 & 0,6613 & 0,00113 & 1,7301 \\
0,00105 & 0,6738 & 0,000795 & 1,7573 & 0,0014 & 0,8646 & 0,001125 & 1,7520
\end{tabular}

Tabla III. Valores de MVP para distintas longitudes de grieta por fatiga en muestras compactas de tracción

Table III. MVP values for different fatigue crack length in compact specimens

\begin{tabular}{lllllllr}
\hline \multicolumn{3}{c}{ Acero 12Cr1MoV } & \multicolumn{5}{c}{ Acero 11Cr1Mo } \\
\hline MVP (V) & Lg (mm) & MVP (V) & Lg (mm) & MVP (V) & Lg (mm) & MVP (V) & Lg (mm) \\
\hline 0,015 & 0,0967 & 0,009 & 1,2472 & 0,021 & 0,0873 & 0,014 & 0,9758 \\
0,014 & 0,2884 & 0,0085 & 1,3430 & 0,02 & 0,1942 & 0,013 & 1,1382 \\
0,013 & 0,4802 & 0,008 & 1,4389 & 0,019 & 0,3066 & 0,012 & 1,3137 \\
0,012 & 0,6719 & 0,0075 & 1,5348 & 0,018 & 0,4251 & 0,011 & 1,5043 \\
0,011 & 0,8637 & 0,007 & 1,6307 & 0,017 & 0,5504 & 0,01 & 1,7132 \\
0,01 & 1,0554 & 0,0065 & 1,7265 & 0,016 & 0,6832 & 0,0099 & 1,7352 \\
0,0095 & 1,1513 & 0,0064 & 1,7457 & 0,015 & 0,8247 & 0,0098 & 1,7575 \\
\hline
\end{tabular}




\section{CONCLUSIONES}

- En este trabajo se ha desarrollado una valoración de los parámetros de la señal del RMB que varían con distintos niveles de deformación plástica y, en consecuencia, con las longitudes de grieta en muestras compactas de tracción preagrietadas por fatiga, fabricadas con aceros utilizados en la industria termoenergética a nivel mundial.

- También se ha demostrado que en las PCT como las aquí empleadas, las grietas se incuban, por regla general, con DP entre $0,75 \%$ y $1 \%$, y que los valores medios de la señal del RMB (Vrms) y el máximo voltaje de pico (MVP) decrecen con el incremento de la deformación plástica de la entalla de la grieta.

- Se obtienen las modelos de correlación entre MVP, Vrms y DP, y Lg y DP, respectivamente, los cuales son combinados para obtener la relación entre longitud de grieta y parámetros del RMB, lo que posibilita evaluar in situ la longitud de la grieta de las PCT.

- Por otra parte, a fin de chequear el momento más exacto de incubación de la grieta, se evalúan en conjunto las densidades espectrales para frecuencias en el orden de $10^{4} \mathrm{~Hz}$, la forma de la envolvente de la señal y que los valores de Vrms y MVP estén próximos a $16,55 \mathrm{mV}$ y $15,5 \mathrm{mV}$ en el $12 \mathrm{Cr} 1 \mathrm{MoV}$, y $23,68 \mathrm{mV}$ y $21,85 \mathrm{mV}$ en el $11 \mathrm{Cr} 1 \mathrm{Mo}$, respectivamente.

- Todo ello contribuye a perfeccionar, en aras de ganar tiempo y calidad de grietas, el proceso de preagrietamiento previo el ensayo de velocidad de propagación de grietas en muestras compactas de tracción.

\section{REFERENCIAS}

[1] W. Soonho, H. Yangki, C. Kyungshik y L. Jaedo, Key Engineering Materials 27 (2004) 70-74.

[2] J. Storesund, B. Kjeld y Z. Weilin, International Journal of Pressure Vessels and Piping 83 (2006) 875-883.

[3] J. Zhao, S. Han, H. Gao y L. Wang, International Journal of Pressure Vessels and Piping 81 (2004) $757-760$.

[4] A. Baltusnikas, R. Levinskas y I. Lukosiute, Material Science (Medžiagotyra) 13 (2007) 286-292.

[5] A. Baltusnikas, R. Levinskas y I. Lukosiute, Material Science (Medžiagotyra) 14 ( 2008) 210-214.
[6] H. Andersson, C.M. Rolf y B. Sandstrom, International Journal of Pressure Vessels and Piping 78 (2001) 749-755.

[7] K. Bong Yoon, P. Tae Gyu y S. Ashok, International Journal of Pressure Vessels and Piping 80 (2003) 465-479.

[8] K. Wsamer, K.M. Nikbin y G.A. Webster, International Journal of Pressure Vessels and Piping 80 (2003) 489-498.

[9] L. Witek, Engineering Failure Analysis 10 (2009) 122-127.

[10] ASTM E1457-00, Standard test method for measurement of creep crack growth rates in metals, 2000.

[11] F. de los Reyes Rodríguez, Tesis doctoral, Universidad de Oriente, Cuba, 2012, p. 106.

[12] H. Barkhausen, Physik. Z. 20 (1919) 401.

[13] S. Yamaura, Y. Furuya y T. Watanabe, Acta Mater. 49 ( 2001) 3.019-3.027.

[14] G. Bertotti y A. Montorsi, J. Magn. Magn. Mater. 83 (1990) 214-216.

[15] M.F. de Campos, F.A. Franco, R. Santos, F.S. da Silva, S.B. Ribeiro, J.F. Lins y L.R. Padovese, Joint European Magnetic Symposia (JEMS 2011), J. Spalek, Ed. Publishing Ltd. Bristol, 2011.

[16] T. Inaguma, H. Sakamoto y M. Hasegawa, J. Appl. Phys. 111 (2012) 063903.1-063903.9.

[17] I. Altpeter, Journal of Nondestructive Evaluation 15 (1996) 45-60.

[18] L.Piotrowski, B. Augustyniaka, M. Chmielewskia e I. Tomáša, J. Magn. Magn. Mater. 321 (2009) 2.331-2.335.

[19] C.G. Stefanita, D.L Atherton y L. Clapham, Acta Mater. 48 (2000) 3.545-3.551.

[20] C.G. Stefanita, L. Clapham y D.L. Atherton, Review of Progress in Quantitative Nondestructive Evaluation, Vols, 19 a and 19 b, Ed. Thompson, Canadá, 2000, pp. 1.541-1.548.

[21] O. Stupakov y I.Tomas, NDT \& E International 39 (2006) 554-561.

[22] M.J. Sablik, J. Appl. Phys. 89 (2001) 5.6105.613.

[23] O. Stupakov, J. Pal'ac, I. Tomáša, J. Bydžovskýc y V. Novák, Mater. Sci. Eng. A 462 (2007) 351-354.

[24] F. Beck, R.C. Gomesa, K.D. Sossmeiera, F. Bohnb y M. Carara, J. Magn. Magn. Mater. 323 (2011) 268-271.

[25] W. Grosse-Nobis, J. Magn. Magn. Mater. 4 (1977) 247-253.

[26] G. Manson y G. Hoffmann, J. Phys. D-Appl. Phys. 5 (1972) 1.389.

[27] V. Moorthy, B.A. Shawa, P. Mountfordb y P. Hopkinsb, Acta Mater. 53 (2005) 4.997-5.006. 